\title{
Registration of visual impairment due to diabetic retinopathy in a subpopulation of Cambridgeshire
}

\author{
Patel Gordon-Bennett \\ Aseema Misra ${ }^{2}$ \\ Wendy Newsom' \\ Declan Flanagan ${ }^{2}$ \\ 'Eye Department, Hinchingbrooke \\ Hospital, Hinchingbrooke Park, \\ Huntingdon, Cambridgeshire, UK; \\ ${ }^{2}$ Eye Department, Addenbrookes \\ Hospital, Hills Road, Cambridge, \\ Cambridgeshire, UK
}

\begin{abstract}
Background/Aims: The UK National Screening Committee (NSC) has set 18 standards for diabetic retinopathy (DR) screening services in England and Wales, the first of which is to reduce new visual impairment (VI) due to DR by $10 \%$ within 5 years. This study examined the incidence of VI due to DR in Cambridgeshire (City, South, and Huntingdonshire) in order to establish a baseline rate of VI registration.
\end{abstract}

Methods: A retrospective review of all certificates of visual impairment (CVI) for 2004 and 2005 was conducted. Hospital records of patients registered due to DR were reviewed to ascertain conformity to NSC Standards. The incidence of VI registration due to DR was calculated.

Results: The number of registrations predominantly due to DR was $18 ; 13$ visually impaired and 5 with severe VI. The rates of VI and severe VI predominantly due to DR were 17.1 and 6.5 per million per year, respectively. The VI and severe VI registration rates in the diabetic population were 600 and 230 per million per year, respectively.

Conclusion: The severe VI registration rate due to DR lies within the national standard. The VI registration rate exceeds 1990-1991 national standards but lies within 1999-2000 national figures.

Keywords: diabetic retinopathy, visual impairment, severe visual impairment, registration, certification of visual impairment

\section{Introduction}

The World Health Organization has made prevention of visual impairment (VI) an international priority. In order to prevent VI, data on incidence and causes of VI need to be obtained. From this data, priorities for prevention, treatment, and management can be identified, and strategies and resources allocated appropriately. For many years, diabetes has been a leading cause of VI in many countries, and still takes a major toll on VI.

In England and Wales, the UK National Screening Committee for Diabetic Retinopathy has set 18 service objectives and quality assurance standards for diabetic retinopathy screening services. ${ }^{1,2}$ The first service objective is to reduce new blindness due to diabetic retinopathy (DR). ${ }^{2}$ It stipulates that local services will need to prospectively audit both certifications of visual impairment (CVI) and incidence of VI predominantly due to DR in order to establish a baseline. The standard acceptable annual registration rate for severe VI and VI due to DR is 9.5 and 9.3 per million per year for England and Wales, respectively. These figures have been derived from national data in 1990-1991. ${ }^{3}$ The minimum standard achievable is a $10 \%$ reduction in severe VI and VI registration rate within 5 years of the start of the screening program. The desirable standard is $40 \%$ reduction.

From 2006 and annually thereafter, DR screening programmes in England and Wales will be required to submit an annual report to the National Screening Programme, containing general information about the service offered and information to support 
an assessment against the Service Objectives and Quality Assurance Standards for the programme. All programmes in England and Wales will be required to use a report template in order to facilitate the collation and comparison of data. ${ }^{4}$ Locally derived data on new VI should be included in the annual report submitted to the National Screening Programme.

Prior to 2005, screening for DR in Cambridgeshire was largely hospital-based, with patients being referred by their general practitioners, diabetic physicians, or optometrists directly to ophthalmologists at either Addenbrookes or Hinchingbrooke Hospital. Optometrists have recently been trained to perform optometric based screening, and to refer patients in a timely manner with maculopathy (M1), preproliferative retinopathy (R2), and proliferative retinopathy (R3).

In November 2003, the CVI replaced the BD8 form in England. The new forms allowed space to record the patient's visual function. Table 1 shows the definitions of severe VI and VI. These apply to the function of the better eye; people with good vision in one eye are not eligible for certification.

This is the first study to obtain the severe VI and VI registration rates predominantly due to DR in south Cambridgeshire, Cambridge city, and Huntingdonshire. It was performed to establish a reference point of VI registration due to DR at the beginning of the screening program.

\section{Methods}

A retrospective review of all CVI for patients with Cambridgeshire post codes over 24 months from January 1, 2004 to December 31, 2005 was conducted. The type of VI, cause of VI and patient's age were noted for each form. An attempt was made to validate the certificates of VI with the central list of all new registrations of VI held by Cambridgeshire Social Services.

Population data was obtained from Census 2001 and Cambridgeshire primary care trusts to determine the total and diabetic populations of South Cambridgeshire, Cambridge city and Huntingdon. We had to exclude East Cambridgeshire and the Fenlands as patients from these areas attend other local district general hospitals eg, Queen Elizabeth Hospital King's Lynn for VI assessment and registration. The annual registration rates of severe VI and VI due to DR were calculated per million population per year. In addition, the rate per million diabetic patients and prevalence of diabetes were calculated.

\section{Results}

From January 1, 2004 to December 31, 2005, there were 367 CVI for patients from Cambridgeshire, Cambridge city, and Huntingdonshire. Two forms were incomplete (the type of VI was not stated). Of the 365 completed forms there were 156 severe VI and 209 VI registrations. Some patients had more than one cause of VI. Each cause was noted which resulted in the number of causes (384) exceeding the number of registration forms (365). The leading causes of VI registration were age-related macular degeneration in 224 or $61.4 \%$ of patients, glaucoma (43 or $11.8 \%$ ), and optic neuropathy (27 or $7.4 \%$ ), (Table 2).

According to Census 2001, the total population of South Cambridgeshire, Cambridge City and Huntingdonshire is 395,933. The number of registrations predominantly due to DR was $18 ; 13$ visually impaired and 5 with severe VI. The rates of severe VI and VI registration predominantly due to DR were 17.1 and 6.5 per million per year, respectively. The diabetic population of South Cambridgeshire (2729), Cambridge City (3361) and Huntingdonshire (4752) in June 2005 was 10,842 , and the prevalence of diabetes in this population of Cambridgeshire is calculated to be $2.74 \%$. The severe VI and VI registration rates in the diabetic population were 230 and 600 per million population with diagnosed diabetes per year, respectively. These results are summarized in Table 3.

\section{Discussion}

In our study, DR was the fifth leading cause of registered VI, superseded by age-related macular degeneration, glaucoma, optic neuropathy, and visual cortex disorder in that order.

Table I Definitions of severe visual impairment and visual impairment used in the UK (apply to the better eye)

\begin{tabular}{ll}
\hline Severe visual impairment & Visual impairment \\
\hline Worse than $3 / 60$ (corrected visual acuity) & $3 / 60$ or $6 / 60$ with full visual field \\
or & or \\
Worse than $6 / 60$ with very contracted visual fields & $6 / 24$ or worse with moderate constriction of visual field \\
or & or \\
$6 / 60$ or above with a very contracted visual field especially & $6 / 18$ or better with gross visual field defects \\
\hline
\end{tabular}


Table 2 Causes of certification of visual impairment (VI) in Cambridgeshire (City, South, and Hunts), 2004-2005

\begin{tabular}{|c|c|c|c|c|c|c|c|}
\hline $\begin{array}{l}\text { Cause of visual } \\
\text { Impairment (VI) }\end{array}$ & $\begin{array}{l}\text { Number of } \\
\text { occurrences }\end{array}$ & $\begin{array}{l}\text { Percentage of } \\
\text { patients } n=365\end{array}$ & VI & Severe VI & $0-15$ years & 15-64 years & $65+$ years \\
\hline ARMD & 224 & $61.4 \%$ & 125 & 99 & 0 & 2 & 222 \\
\hline Glaucoma & 43 & $11.8 \%$ & 27 & 16 & 0 & 2 & 41 \\
\hline Retinitis pigmentosa & 12 & $3.3 \%$ & 5 & 7 & 0 & 7 & 5 \\
\hline Diabetic retinopathy & 18 & $4.9 \%$ & 13 & 5 & 0 & 3 & 15 \\
\hline Visual cortex disorder & 19 & $5.2 \%$ & 15 & 4 & I & 5 & 13 \\
\hline Optic neuropathy & 27 & $7.4 \%$ & 18 & 9 & 3 & 12 & 12 \\
\hline Retinal detachment & 7 & $2 \%$ & 5 & 2 & 0 & 2 & 5 \\
\hline Cause unstated & 12 & $3.3 \%$ & 6 & 6 & 4 & 1 & 7 \\
\hline Others & 22 & $6.0 \%$ & 14 & 8 & 1 & 7 & 14 \\
\hline Total occurrences & 384 & & 228 & 156 & 9 & 41 & 334 \\
\hline Total CVI forms & 365 & & 209 & 156 & 9 & 41 & 315 \\
\hline
\end{tabular}

In persons of working age (16-64 years), the leading cause of VI registration was optic neuropathy followed by retinitis pigmentosa (Table 2). The specific causes of optic neuropathy were not stated in most instances on the CVI form. Most optic neuropathies were congenital or secondary to demyelination and did not include diabetic or glaucomatous optic neuropathy. Further studies are needed to clarify the types of optic neuropathy, by examining the patient records. A comparison of the demographic profile of our study population with that of England and Wales reveals a younger age profile (Table 4). There are $0.8 \%$ less persons aged 75 years and over, and $0.6 \%$ more persons in the $0-15$ year age group of Cambridge city, South Cambridgeshire, and Huntingdonshire. These differences in age profile may contribute to the differences observed.

Three diabetic patients were of working age (16-64 years). From our study, DR does not appear to be the leading cause of CVI in the working age group in this population. This finding is contrary to that of Bunce and colleagues ${ }^{5}$ who found that
DR was the leading cause of VI in the working age group in England and Wales in 1999-2000.

The severe VI registration rate of 6.5 per million per year meets the national standard of 9.5 per million per year. The VI registration rate was 17.1 per million per year, and almost doubles the current national standard of 9.3 per million per year. However, new data on national VI registration has emerged from a study carried out by Bunce and colleagues ${ }^{5}$ in 1999-2000. In this study the rate of certification of VI due to DR was 38.4 per million per year, compared to 19.9 per million per year in 1990-1991.,,6 These rates have doubled over the nine years, partly explained by increased VI registration rates during the time interval 1991-1999, an aging population, and increasing prevalence of Type 2 and Type 1 diabetes. ${ }^{5}$ Given these new figures, we recommend that the national standards of severe VI and VI registration rates be revised. A severe VI registration rate of 12 per million population per year has been reported for Fife from 1990-1999, ${ }^{7}$ and a severe VI registration rate of 10 per million

Table 3 Summary of registrations of visual impairment (VI) due to diabetic retinopathy (DR) in Cambridgeshire (City, South, and Hunts) (2004-2005)

\begin{tabular}{ll}
\hline Number of registrations with severe VI due to DR & $5(3.2 \%$ of severe VI registrations) \\
Number of registrations with partialVI due to DR & $13(6.2 \%$ of partial VI registrations) \\
Number of registrations with VI due to DR in working & $0 \%$ of severe VI registrations \\
age group (I6-64 years) & $1.4 \%$ of partialVI registrations \\
Severe VI registration rate due to DR & 6.5 per million population per year \\
PartialVI registration rate due to DR & 17.1 per million population per year \\
Number of diabetics in Cambs City, South, Hunts 2005 & 10,842 \\
Prevalence of diabetes & $2.74 \%$ \\
Severe VI registration rate in diabetics & 230 per million diabetics per year \\
Partial VI registration rate in diabetics & 600 per million diabetics per year \\
\hline
\end{tabular}


Table 4 Demographics of Cambridgeshire (Census 200I)

\begin{tabular}{llll}
\hline & $\begin{array}{l}\text { Cambridge City, South } \\
\text { and Huntingdonshire }\end{array}$ & Cambridgeshire & England and Wales \\
\hline Total population & 395,933 & 552,658 & $52,041,916$ \\
$\quad$ Male & $49.7 \%$ & $49.5 \%$ & $48.7 \%$ \\
Female & $50.3 \%$ & $50.5 \%$ & $51.3 \%$ \\
Ethnic group & & & $90.0 \%$ \\
White & $95.0 \%$ & $95.9 \%$ & $1.5 \%$ \\
Mixed & $1.2 \%$ & $1.0 \%$ & $4.5 \%$ \\
Asian & $1.7 \%$ & $1.3 \%$ & $3.0 \%$ \\
Black & $0.7 \%$ & $0.6 \%$ & $1.0 \%$ \\
Chinese/Other & $1.4 \%$ & $1.2 \%$ & $18.7 \%$ \\
Age (years) & & & $74.0 \%$ \\
$0-15$ & $19.3 \%$ & $19.4 \%$ & $7.3 \%$ \\
16-74 & $74.2 \%$ & $73.6 \%$ & $7.0 \%$ \\
$75+$ & $6.5 \%$ & & \\
\hline
\end{tabular}

Note: Percentages given are proportions of the total population of the column.

per year and a VI registration rate of 24 per million per year was reported for Leeds for $2002 .{ }^{8}$

The prevalence of diabetes in South Cambridgeshire, Cambridge city, and Huntingdonshire (2.74\%) is less than the national prevalence estimated as $4.26 \% .{ }^{9}$ The prevalence in Leeds is $2.9 \%{ }^{8}$ The region of Cambridgeshire studied has about $5 \%$ more Whites than the ethnic profile of England/Wales, (Table 4). Likewise, our studied region has $2.8 \%$ less Asians and 2.3\% less Blacks than England/Wales. This difference might account for the slightly lower prevalence of diabetes than the national prevalence, as there is well documented evidence of an increased prevalence of diabetes in Asians and Blacks. ${ }^{10,11}$ The severe VI and VI registration rates per million diabetic patients per year were 230 and 600 respectively. These rates are lower than the corresponding rates in the diabetic population of Leeds of 337 and 817, respectively. ${ }^{8}$ The rates of registration in our study are also lower than the severe VI registration rate of 640 per million diabetics per year reported for Fife between 1990-1999, ${ }^{7}$ and similar to the VI registration rate of 530 per million diabetics per year calculated for Tayside in $1998 .{ }^{12}$ This interregional variation may be related to differing population profiles. ${ }^{13,14}$

Addenbrookes Hospital is a tertiary referral hospital in Cambridge city and Hinchingbrooke Hospital is a secondary care centre which serves as a district general hospital for Huntingdonshire and other surrounding subpopulations of Cambridgeshire. We questioned whether our relatively low rates of registration of VI due to diabetes were due to the nature of these hospitals. Geographically, Addenbrookes Hospital is the closest hospital to patients living in Cambridge city and South Cambridgeshire and being a tertiary referral centre, it would serve all patients in Cambridge in addition to receiving referrals from outside Cambridge. Very few diabetic patients would be referred elsewhere for treatment. Our results were cross-checked with the relevant Primary Care Trusts and both sets of data correlated very well. The Primary Care Trusts had no record of any patient living in the study population, who was registered visually impaired outside of Addenbrookes or Hinchingbrooke Hospitals or from the private sector in the study period. The possibility of a large number patients being registered visually impaired in the private sector is unlikely.

It is well known that there are limitations in using VI registration data to study rates of VI. ${ }^{15,16,17}$ VI registration data are hospital-based, not population-based. ${ }^{5}$ The patient must access the hospital eye service in order to be seen by a consultant ophthalmologist. Between $43 \%$ and $58 \%$ of eligible outpatients are said to remain unregistered even after consultation with an ophthalmologist, ${ }^{15,16}$ and up to $40 \%$ of registered patients are inappropriately registered. ${ }^{15}$ Patient attitudes to registration process (patients are entitled to refuse the offer), and medical attitudes to registration (there is no statutory requirement for it to be offered) also affect registration rates. ${ }^{5,18}$ There is often a delay between onset of certifiable visual loss and offer of registration. ${ }^{5}$ Studies have shown that certification of VI is biased towards severe visual loss, permanent, nontreatable causes, and those that affect central rather than peripheral vision. ${ }^{15,17}$ Ethnic minorities are less likely to undergo registration. ${ }^{16,19}$ A prospective audit of 
incident VI would be more useful. Despite these limitations, data on VI registration rates may be useful as estimates of incidence of VI and to establish a baseline for DR screening programmes.

\section{Conclusions}

From our study it would appear that registration of severe VI secondary to DR is uncommon and does not appear to be the main cause of VI registration in the working age group in this population of Cambridgeshire. The severe VI registration rate due to DR lies within the 1990-1991 national standard. The VI registration rate exceeds 1990-1991 national standard but lies within 1999-2000 national figures. These rates form a baseline for the region, to which future rates can be compared. We are unlikely to be able to achieve a lower rate of severe VI registration from DR. Improvement in VI due to DR may depend on improving other aspects of diabetic care, such as control of blood glucose and blood pressure, particularly before retinopathy occurs, and timely referral of patients with sight-threatening retinopathy.

\section{Acknowledgments}

We thank the visual impairment liaison coordinator Lynette Gayfer, the secretarial staff and receptionists of Addenbrookes and Hinchingbrooke Hospitals, the primary care trusts within Cambridgeshire, and Jan Suart-Menteath of Cambridgeshire City Council.

\section{Disclosure}

The authors report no conflicts of interest in this work. No funding was received for this study.

\section{References}

1. UK National Screening Committee. Essential elements in developing a diabetic retinopathy screening programme, Workbook 3. Nov. 2004.

2. Garvican L, Scanlon P. Quality Assurance for the National Screening Programme for Sight-threatening Diabetic Retinopathy: Development of a Set of Key Quality Assurance Standards. Sept. 2003. Available from: http://www.nscretinopathy.org.uk/resources/FinalQAStdsSept03.doc.
3. Evans JR. Causes of blindness and partial sight in England and Wales 1990-91, Studies on Medical and Population Subjects Reports No. 57 HMSO: London, 1995.

4. National Screening Programme for Diabetic Retinopathy. Template for Annual Report - Release 3. July 20, 2005.

5. Bunce C, Wormald R. Leading causes of certification for blindness and partial sight in England and Wales. BMC Public Health. 2006;6:58.

6. Evans JR, Rooney C, Ashwood F, et al. Blindness and partial sight in England and Wales: April 1990-March 1991. Health Trends. 1996;28:5-12.

7. Cormack TG, Grant B, Macdonald MJ, et al. Incidence of blindness due to diabetic eye disease in Fife 1990-9. Br J Ophthalmol. 2001;85:354-6.

8. Kumar N, Goyder E, McKibbin M. The incidence of visual impairment due to diabetic retinopathy in Leeds. Eye. 2006;20:455-9.

9. National Diabetes Audit. Key findings about the quality of care for people with diabetes in England. Report for the audit period 2003/04.

10. Mather HM, Keen H. The Southall Diabetes Survey: prevalence of known diabetes in Asians and Europeans. Br Med J (Clin Res Ed). 1985;291:1081-4.

11. Cowie CC, Rust KF, Byrd-Holt DD, et al. Prevalence of diabetes and impaired fasting glucose in adults in the U.S. Population: National Health and Nutrition Examination survey 1999-2002. Diabetes Care. 2006;29:1263-8.

12. Bamashmus MA, Matlhaga B, Dutton GN. Causes of blindness and visual impairment in the West of Scotland. Eye. 2004;18:257-61.

13. Hayward LM, Burden ML, Burden AC, et al. What is the prevalence of visual impairment in the general and diabetic populations: are there ethnic and gender differences? Diabet Med. 2002;19:27-34.

14. Pardhan S, Gilchrist J, Mahomed I. Impact of age and duration on sight-threatening retinopathy in South Asians and Caucasians attending a diabetic clinic. Eye. 2004;18:233-40.

15. Barry RJ, Murray PI. Unregistered visual impairment: is registration a failing system? Br J Ophthalmol. 2005;89:995-8.

16. Robinson R, Deutsch J, Jones HS, et al. Unrecognised and unregistered visual impairment. Br J Ophthalmol. 1994;78:736-40.

17. Bunce C, Evans J, Fraser S, et al. BD8 certification of visually impaired people. Br J Ophthalmol. 1998;82:72-6.

18. Canavan YM, Jackson AJ, Stewart A. Visual impairment in Northern Ireland. Ulster Med J. 1997;66:92-5.

19. Pardhan S, Mahomed I. The clinical characteristics of Asian and Caucasian patients on Bradford's Low Vision Register. Eye. 2002;16:572-6. 
